\title{
Modified waste egg shell derived bifunctional catalyst for biodiesel production from high FFA waste cooking oil: a review
}

\begin{abstract}
Global energy crisis are as a result of gradual depletion of fossil fuel reserves, coupled with population growth in developing countries. Besides, fossil fuels are not environmentally benign as they are associated with problems, i.e. global warming, high toxicity and non biodegradability, hence it is considered as non sustainable source of energy. Without doubt, biofuel-based energy is a promising long-term energy source that can reduce the over dependence on fossil fuels as a result of feedstocks availability and renewability. However, biodiesel production from vegetable oil using the traditional homogeneous catalytic system is no longer defensible by industries in the near future, particularly due to food-fuel rivalry and ecological problems related to the conventional homogeneous catalytic system. This review presents a comprehensive step by step process of converting waste cooking oil (WCO) to biodiesel, using modified waste egg shell catalyst. The modified waste egg shell derived bifunctional catalyst could easily be removed from the fatty acid methyl esters (FAME) with limited environmental effects. The new modified catalytic system is able to convert the high free fatty acid (FFA) content waste cooking oil to FAME efficiently under moderate reaction conditions. Utilization of waste cooking oil as a feedstock for biodiesel production will reduce the food security issues that stem the biodiesel production from food-grade oil. Moreover, it will reduce the total production cost of the FAME due to its low cost. The major objective of this article is to demonstrate the current state of the use of heterogeneous bifunctional acid/base catalyst to produce biodiesel from green and nonedible waste cooking oil. At the end of the article, perspectives and future developments are also presented.
\end{abstract}

Keyword: Fossil fuel; Biofuel; Vegetable oil; High FFA waste cooking oil; Homogeneous catalyst; Heterogeneous catalyst 